\title{
Factores sociales para el uso de alcohol en adolescentes y jóvenes
}

\author{
Laura Débora Acosta ${ }^{1}$ \\ Alicia Ruth Fernández ${ }^{2}$ \\ Sandra Cristina Pillon ${ }^{3}$
}

\begin{abstract}
Esta investigación analiza factores sociales de riesgo y de protección para el consumo excesivo de alcohol a partir de los datos de la Encuesta Nacional sobre prevalencias de consumo de sustancias psicoactivas, en 2008, en la población de jóvenes argentinos de 16 a 24 años. Se realizó análisis bivariado y multivariado. Se observó como factores de riesgo: la edad de inicio (el menor edad tiene mayor riesgo), la región Pampeana, Noroeste y Noreste, el género masculino y el provenir de hogares con necesidades básicas insatisfechas. Entre los factores protectores identifica: inactividad, no haber tenido posibilidad de probar drogas ilícitas, no sentir deseos de probar drogas ilícitas, y no haber consumido: tabaco, tranquilizantes, cocaína y pasta base - alguna vez en la vida. Los resultados aportan información útil para la formulación de programas preventivos para la población adolescente y joven.

Descriptores: Factores de Riesgo; Adolescente; Consumo de Bebidas Alcohólicas/Estadística \& Datos Numéricos.
\end{abstract}

\footnotetext{
${ }^{1}$ Licenciada en Nutrición, Becaria de Investigación, Escuela de Salud Pública, Facultad de Ciencias Médicas, Universidad Nacional de Córdoba, Argentina. E-mail: laudeac@hotmail.com.

2 Doctora en Medicina, Profesor Titular, Escuela de Salud Pública, Facultad de Ciencias Médicas, Universidad Nacional de Córdoba, Argentina. E-mail: rfernandez@fcm.un.edu.ar.

${ }^{3}$ Enfermera, Doctora en Enfermería. Profesor Asociado, Escola de Enfermagem de Ribeirão Preto, Universidade de São Paulo, Centro Colaborador de la OMS para el Desarrollo de la Investigación en Enfermería, SP, Brasil. E-mail: pillon@eerp.usp.br.
}

Correspondencia:

Sandra Cristina Pillon

Universidade de São Paulo. Escola de Enfermagem de Ribeirão Preto

Departamento de Enfermagem Psiquiátrica e Ciências Humanas

Av. dos Bandeirantes, 3900

Bairro: Monte Alegre

CEP: 14040-902 Ribeirão Preto, SP, Brasil

E-mail: pillon@eerp.usp.br 


\section{Fatores sociais para o uso de álcool em adolescentes e jovens}

Este estudo analisa os fatores sociais de risco e proteção para o uso excessivo de álcool, a partir dos dados da "Pesquisa nacional sobre a prevalência do uso de substâncias psicoativas, em 2008", na população de jovens argentinos, de 16 a 24 anos. A análise bivariada e análise multivariada foram utilizadas. Observou-se, como fatores de risco para a idade de início (quanto mais jovem, maior o risco), a região dos Pampas, Noroeste e Nordeste, o sexo masculino, provenientes de famílias com necessidades básicas insatisfeitas e idade de início. Entre os fatores de proteção encontram-se a ociosidade, não ter oportunidade de experimentar drogas ilícitas, não se sentir tentado a experimentar drogas ilícitas e não ter usado o tabaco, ou tranquilizantes, ou cocaína, ou base de cocaína, uma vez na vida. Os resultados fornecem informações úteis para o desenvolvimento de programas de prevenção na população adolescente e jovens.

Descritores: Fatores de Risco; Adolescente; Consumo de Bebidas Alcoólicas/Estatística \& Dados Numéricos.

\section{Social Risk Factors for Alcohol Use Among Adolescents and Youth}

This study examines the social risk and protection factors for excessive alcohol use, based on the data from the "National Survey on the prevalence of substance abuse in 2008", in a population of young Argentines, from 16 to 24 years of age. Bivariate and multivariate analyses were used. The following risk factors were observed: age at onset (the younger, the higher the risk); the Pampas region, Northwest and Northeast; males; from families with unsatisfied basic needs. Protective factors include idleness, no opportunity to try out illicit drugs, not being tempted to experiment with illicit drugs and no previous use of tobacco, or tranquilizers, or cocaine or cocaine-based substances in the lifetime. The results provide useful information to develop prevention programs for adolescents and young people.

Descriptors: Risk Factors, Adolescent; Alcohol Drinking /Statistics \& Numerical Data.

\section{Introducción}

Son múltiples las razones por las cuales los jóvenes son vulnerables al consumo excesivo de alcohol. Esta alta vulnerabilidad, es producto de un conjunto de riesgos que surgen debido al proceso de desarrollo y a los cambios particulares que ocurren en esta etapa en la esfera neurológica, cognitiva y social(1). En la esfera social, la adolescencia supone una fase de desarrollo donde el individuo configura su identidad frente al grupo de pares, lo cual lo puede llevar a adoptar modelos de comportamiento arriesgados, lo que incluye el consumo de drogas lícitas e ilícitas ${ }^{(2)}$.

Por otra parte, el consumo excesivo de alcohol, hoy en día, es tolerado por la sociedad, incluso aprobado. A pesar que no sólo la dependencia del alcohol sino que también otras formas de consumo son nocivas para la salud del individuo, la mirada de la sociedad frente al consumo y la dependencia es diferente ${ }^{(3-4)}$.

Internacionalmente puede observarse un fenómeno actual en el patrón de consumo de alcohol en los jóvenes, por la cual muchos de ellos beben hasta "estar ebrios". Estudios han demostrado una alta prevalencia de ebriedad así como una tendencia a estar cada vez "más ebrios", lo cual involucra el consumo de bebidas destiladas entre la población joven(5).

El término usado en la actualidad para este tipo de consumo es el de "binge drinking" (consumo episódico excesivo de alcohol), el cual es definido como el consumo de 5 o más tragos en una ocasión, incluso cuando la ingesta promedio no es excesiva ${ }^{(6)}$. Este es un patrón de consumo de alcohol prevalente en los adolescentes y jóvenes ${ }^{(7)}$. 
El "binge drinking" puede incrementar el riesgo de enfermedades cardiovasculares incluyendo isquemia, angina y accidente cardiovascular ${ }^{(8)}$. Las edades jóvenes, el "binge drinking" ha sido asociado con accidentes, suicidios, relaciones sexuales sin protección y uso de drogas ilícitas ${ }^{(9)}$.

El presente artículo tiene como propósito analizar factores sociales de riesgo y de protección para el consumo excesivo de alcohol a partir de los datos de la "Encuesta Nacional sobre prevalencias de consumo de sustancias psicoactivas 2008 en la población de jóvenes argentinos.

\section{Método}

El presente estudio es de naturaleza cuantitativa, analítica y transversal. El Universo del estudio estuvo constituido por adolescentes y jóvenes de ambos sexos de 16 a 24 años de las regiones Gran Buenos Aires, Pampeana, Noroeste, Cuyo y Patagonia de la Argentina, en el año 2008.

La muestra del presente estudio se basa en datos secundarios (EnPreCoSP-2008) de la encuesta nacional realizada por el Instituto Nacional de Estadísticas y Censos (INDEC) y el Ministerio de Salud de la Nación(10). El muestreo fue probabilístico, de múltiples etapas y estratificado de acuerdo al tamaño de la aglomeración en función de viviendas, jurisdicción del país de pertenencia y ubicación geográfica, datos de actividad económica y tasa de crecimiento intercensal. Los hogares seleccionados son formados por dos bloques: uno que refiere a los datos sobre condiciones habitacionales, aspectos sociodemográficos, y educativos de todas las personas que conforman el hogar; y otro destinado a relevar datos sociodemográficos, educativos y sobre la situación laboral y de salud general, así como el consumo de sustancias psicoactivas. Este bloque se aplicó a un único integrante seleccionado al azar dentro del hogar. La muestra final estuvo constituida por 34.203 individuos, de los cuales $6.736(20 \%)$ se encontraban dentro del intervalo de edad de 16 a 24 años, constituyendo la muestra del presente trabajo.

Las variables de la encuesta y consideradas en el análisis de este estudio fueron: Región del país, Características del hogar al que pertenece la persona seleccionada: Tamaño del hogar, Tipo de hogar, Ingreso total mensual del hogar e Indicadores de Necesidades Básicas Insatisfechas del hogar; Características del Jefe del Hogar: Sexo, Edad, Situación Conyugal, Nivel de Instrucción, Cobertura de Salud y Situación Laboral;
Características de los adolescentes y jóvenes: Relación de Parentesco del seleccionado con el Jefe, Sexo, Edad, Situación Conyugal, Nivel de Instrucción, Cobertura de Salud y Situación Laboral; Autopercepción de salud acceso y entorno de los adolescentes y jóvenes; Prevalencia del uso en la vida de sustancias psicoactivas: Tabaco, Tranquilizantes, Estimulantes, Marihuana, Cocaína, Pasta base, Éxtasis, Inhalables, Otras drogas alcohol: Edad a la que consumió alcohol por primera vez, frecuencia de consumo, uso en la vida, año y mes, consumo abusivo y consumo episódico excesivo. El Consumo episódico excesivo de bebidas alcohólicas constituyó la variable dependiente. La misma resulta cuando el encuestado consumió 5 o más tragos al menos una vez en los últimos 30 días de los respectivos tipos de bebidas, entre semana o en el fin de semana, denominado como "binge drinking".

Respecto a las consideraciones éticas, la base de datos de la encuesta EnPreCoSP-2008 fue obtenida de la Dirección de Estadísticas de la Provincia de Córdoba. La base de datos posee un código de identificación para cada encuestado, el cual fue confidencialmente resguardado en el proceso de investigación. Asimismo, esta investigación ha sido aprobada por el Comité de Ética del Hospital Nacional de Clínicas de la ciudad de Córdoba, Argentina.

El análisis descriptivo de las variables fue efectuado a partir de medidas de resumen. Se efectuó un análisis bivariado a través de datos categóricos (Prueba de Chi cuadrado o prueba de Fisher) obteniéndose Odds Ratio (OR) e Intervalos de Confianza (IC); o prueba T Student para muestras independientes, según la naturaleza de la variable independiente. Además se realizó un Análisis de Regresión Logística, incluyendo en el modelo todas las variables que presentaron asociación estadísticamente significativa en el análisis bivariado. En todos los casos se estableció un nivel de significación de $p<0,05$. Se utilizó el programa estadístico SPSS versión 15.0 para Windows.

\section{Resultados}

En relación a la región del país, el $5 \%$ pertenece a la zona del Gran Buenos Aires, el 18,6\% a la región Pampeana, el 28,5\% a la región Noroeste, el 19,0\% a la región Noreste, el 12,1\% a la región Cuyo y el 16,8\% a la región Patagónica. En relación a las características del hogar de los/as adolescentes y jóvenes, la media del número de miembros del hogar fue de 4,38 personas $\mathrm{DE} \pm 2,21$. 
El $58,2 \%$ de la población bajo estudio vive en hogares que perciben ingresos menores o iguales a 1.500 pesos (400 dólares). Las demás características de la vivienda y las características del jefe de hogar, se resumen en la Tabla 1 . Se observa que el tipo de hogar más frecuente fue el multipersonal completo con hijos y sin otros miembros.

Tabla 1 - Características de la vivienda y del jefe de hogar de los adolescentes y jóvenes de 16 a 24 años de Argentina. Encuesta EnPreCoSP-2008 ( $n=6.736)$

\begin{tabular}{|c|c|c|c|}
\hline Características de la vivienda & Categorías & $\mathbf{N}$ & $\%$ \\
\hline \multirow[t]{8}{*}{ Tipo de hogar } & Unipersonal & 375 & 5,6 \\
\hline & Multipersonal conyugal completo sin hijos ni otros miembros & 188 & 2,8 \\
\hline & Multipersonal conyugal completo sin hijos y con otros miembros & 123 & 1,8 \\
\hline & Multipersonal conyugal completo con hijos y sin otros miembros & 2768 & 41,1 \\
\hline & Multipersonal conyugal completo con hijos y con otros miembros & 879 & 13,0 \\
\hline & Multipersonal conyugal incompleto sin otros miembros & 1075 & 16,0 \\
\hline & Multipersonal conyugal incompleto con otros miembros & 722 & 10,7 \\
\hline & Multipersonal no conyugal & 606 & 9,0 \\
\hline \multirow[t]{4}{*}{ Necesidades Básicas Insatisfechas } & Ningún indicador & 5572 & 82,7 \\
\hline & Al menos un indicador & 34 & 0,5 \\
\hline & Al menos dos indicadores & 910 & 13,5 \\
\hline & Tres o más indicadores & 220 & 3,3 \\
\hline \multicolumn{4}{|l|}{ Características del jefe de hogar } \\
\hline \multirow[t]{2}{*}{ Sexo } & Masculino & 4263 & 63,3 \\
\hline & Femenino & 2473 & 36,7 \\
\hline \multirow[t]{3}{*}{ Grupo de edad } & 16 a 34 años & 1766 & 26,2 \\
\hline & 35 a 49 años & 2392 & 35,5 \\
\hline & 50 o más & 2578 & 38,2 \\
\hline \multirow[t]{4}{*}{ Situación conyugal del jefe/a } & Unido Casado/a & 4030 & 59,9 \\
\hline & Separado o Divorciado/a & 929 & 13,8 \\
\hline & Viudo/a & 574 & 8,5 \\
\hline & Soltero/a & 1203 & 17,9 \\
\hline \multirow[t]{5}{*}{ Nivel de instrucción del jefe/a } & Sin instrucción & 135 & 2,0 \\
\hline & Primario completo o incompleto & 2627 & 39,0 \\
\hline & Secundario completo o incompleto & 2434 & 36,1 \\
\hline & Terciario o universitario completo o incompleto y más & 1537 & 22,9 \\
\hline & Educación especial & 3 & 0,0 \\
\hline \multirow[t]{6}{*}{ Cobertura de salud del jefe } & Sólo plan de salud privado o/y otro & 526 & 7,8 \\
\hline & Sólo obra social & 3603 & 53,5 \\
\hline & Obra social y otro no privado & 149 & 2,2 \\
\hline & Sólo servicio de emergencia o Sólo plan o seguro público & 148 & 2,2 \\
\hline & No está asociado a nada & 2224 & 33,0 \\
\hline & $\mathrm{Ns} / \mathrm{Nc}$ & 86 & 1,3 \\
\hline \multirow[t]{3}{*}{ Condición de actividad del jefe/a } & Ocupado & 5065 & 75,2 \\
\hline & Desocupado & 219 & 3,3 \\
\hline & Inactivo & 1452 & 21,6 \\
\hline \multirow[t]{5}{*}{ Categoría ocupacional del jefe/a $(n=5622)$} & Patrón o empleador & 279 & 4,1 \\
\hline & Cuenta propia & 1257 & 18,7 \\
\hline & Asalariado (excluye servicio doméstico) & 3736 & 55,5 \\
\hline & Asalariado (sólo servicio doméstico) & 281 & 4,2 \\
\hline & Trabajador familiar & 69 & 1,0 \\
\hline
\end{tabular}

En relación al indicador Necesidades Básicas Insatisfechas, el total de hogares con algún indicador NBI fue de $17,3 \%$. En cuanto a las características del jefe del hogar, se destaca que el $63 \%$ fueron de sexo masculino, el 73,7\% fueron mayores de 35 años; el 50\% se encontraba unido o casado, el $41 \%$ tenía educación primaria o menos; más de tres cuartas partes se encontraba ocupado y más de la mitad de la población poseía obra social. 
En cuanto a las características de los/as jóvenes y adolescentes de 16 a 24 años, el promedio de edad fue de 20,07 años $D E \pm 2,57$. El resto de las características se resumen en la Tabla 2 . Se destaca que la mayoría fueron hijos/as del jefe de hogar, la mayoría se encontraba soltero/a, el $12,4 \%$ poseía educación primaria o menos, la mayoría poseía obra social y se encuentran inactivos. El mayor porcentaje de la población indicó su salud como buena, y la mayoría no había padecido enfermedad o accidente o había visitado a un profesional de la salud por problemas de angustia, relaciones personales o depresión en los últimos doce meses.

Tabla 2 - Características sociodemográficas, educativas y de salud de los adolescentes y jóvenes de 16 a 24 años de Argentina. Encuesta EnPreCoSP-2008 ( $N=6736)$

\begin{tabular}{|c|c|c|c|}
\hline Variables & Categorías & $\mathbf{N}$ & $\%$ \\
\hline \multirow[t]{9}{*}{ Relación del parentesco con el jefe/a } & Jefe/a & 881 & 13,1 \\
\hline & Cónyuge/pareja & 559 & 08,3 \\
\hline & Hijo/a / hijastro/a & 4283 & 63,6 \\
\hline & Hermano/a & 194 & 02,9 \\
\hline & Suegro/a & 4 & 00,1 \\
\hline & Yerno/nuera & 144 & 02,1 \\
\hline & Nieto/a & 390 & 05,8 \\
\hline & Otro familiar & 161 & 02,4 \\
\hline & Otro no familiar & 120 & 01,8 \\
\hline \multirow[t]{2}{*}{ Sexo } & Hombre & 3228 & 47,9 \\
\hline & Mujer & 3508 & 52,1 \\
\hline \multirow[t]{4}{*}{ Situación conyugal } & Unido o Casado & 1166 & 31,7 \\
\hline & Separado o Divorciado & 40 & 00,6 \\
\hline & Viudo/a & 5 & 00,1 \\
\hline & Soltero/a & 5525 & 82,0 \\
\hline \multirow[t]{5}{*}{ Nivel de instrucción } & Sin instrucción & 19 & 00,3 \\
\hline & Primario incompleto o completo & 1139 & 12,1 \\
\hline & Secundario incompleto o completo & 3872 & 57,5 \\
\hline & Terciario o universitario incompleto o completo y más & 2014 & 29,9 \\
\hline & Educación especial & 17 & 00,3 \\
\hline \multirow[t]{7}{*}{ Cobertura de salud } & Sólo plan de salud privado & 409 & 06,1 \\
\hline & Plan de salud privado y otro & 111 & 01,6 \\
\hline & Sólo obra social & 3034 & 45,0 \\
\hline & Obra social y otro no privado & 112 & 01,7 \\
\hline & Sólo servicio de emergencia & 77 & 01,1 \\
\hline & Sólo plan o seguro público & 91 & 01,4 \\
\hline & No está asociado a nada & 2797 & 41,5 \\
\hline \multirow[t]{3}{*}{ Condición de actividad } & Ocupado & 3043 & 45,2 \\
\hline & Desocupado & 484 & 7,2 \\
\hline & Inactivo & 3209 & 47,6 \\
\hline \multirow[t]{4}{*}{ Autopercepción de salud } & Excelente & 989 & 14,7 \\
\hline & Muy buena & 2190 & 32,5 \\
\hline & Buena & 2954 & 43,9 \\
\hline & Regular o Mala & 603 & 09,0 \\
\hline Accidente en los últimos 12 meses & Sí & 487 & 07,2 \\
\hline Enfermedad en los últimos 12 meses & Sí & 1299 & 19,3 \\
\hline Visita a profesional de la salud en los últimos 12 meses & Sí & 756 & 11,2 \\
\hline
\end{tabular}

En cuanto a las características de acceso y entorno, el $37,4 \%$ refirió conocer a personas que consumen sustancias ilícitas, el $14 \%$ afirmó tener curiosidad por consumir drogas ilícitas, el 33,7\% refirió tener posibilidad de consumir alguna droga ilícita, y el 4,8\% afirmó que probaría drogas ilícitas. En cuanto a la prevalencia 
de vida de consumo de drogas, casi uno de cada dos adolescentes consumieron tabaco alguna vez en la vida (48, 4\%); la droga ilícita más consumida fue la marihuana $8,8 \%$; la prevalencia de vida de tranquilizantes fue de 3,2\%; de de cocaína 1,8\%; de inhalables $1,5 \%$; de medicamentos para adelgazar 1,5\%; de estimulantes $0,7 \%$; de pasta base $0,5 \%$ de éxtasis $0,5 \%$; de opiáceos y anestésicos $0,3 \%$; de consumo de crack $0,04 \%$; otras drogas $0,5 \%$.

En cuanto al consumo de alcohol, la edad promedio en que bebió alcohol por primera vez fue a los 15,8 años $D E \pm 1,97$ años, siendo la edad mínima a los 5 años y la máxima a los 24 años. El resto de las variables relacionadas con el consumo de alcohol se encuentran resumidas en la Tabla 3. Se destaca que la prevalencia de vida de consumo de bebidas alcohólicas es mayor que para cualquier otra sustancia psicoactiva; y casi la mitad de la población posee prevalencia de mes. La bebida más consumida es la cerveza, en especial los fines de semana.

Tabla 3 - Perfil de consumo de alcohol de los adolescentes y jóvenes de 16 a 24 años de Argentina. Encuesta EnPreCoSP-2008 $(\mathrm{N}=6736)$

\begin{tabular}{|c|c|c|c|}
\hline Variable & Categoría & $\mathbf{N}$ & $\%$ \\
\hline Prevalencia de vida de consumo de bebidas alcohólicas & Sí & 5071 & 75,3 \\
\hline Prevalencia del último año de consumo de bebidas alcohólicas & Sí & 4533 & 67,3 \\
\hline Prevalencia del último mes de consumo de bebidas alcohólicas & Sí & 3267 & 48,5 \\
\hline Se emborrachó alguna vez en los últimos 30 días $(n=3267)$ & Sí & 718 & 10,7 \\
\hline \multirow[t]{4}{*}{ Percepción acerca del riesgo de un bebedor de vez en cuando } & Ningún riesgo & 1216 & 18,1 \\
\hline & Riesgo leve o moderado & 3186 & 47,3 \\
\hline & Gran riesgo & 2126 & 31,6 \\
\hline & No sé qué riesgo corre & 208 & 3,1 \\
\hline \multirow[t]{4}{*}{ Percepción acerca del riesgo de un bebedor frecuente } & Ningún riesgo & 33 &, 5 \\
\hline & Riesgo leve o moderado & 485 & 7,2 \\
\hline & Gran riesgo & 6089 & 90,4 \\
\hline & No sé qué riesgo corre & 129 & 1,9 \\
\hline Consumo episódico excesivo de cerveza(n=3267) & Sí & 877 & 13,0 \\
\hline Consumo episódico excesivo de vino & Sí & 282 & 4,2 \\
\hline Consumo episódico excesivo de bebida fuerte $(n=3267)$ & Sí & 144 & 2,1 \\
\hline Consumo episódico excesivo de bebidas alcohólicas entre semana $(n=3267)$ & Sí & 81 & 1,2 \\
\hline Consumo episódico excesivo de bebidas alcohólicas en fin de semana ( $\mathrm{n}=3267)$ & Sí & 1195 & 17,7 \\
\hline Consumo episódico excesivo de total combinado de bebidas alcohólicas ( $n=3267$ ) & Sí & 1205 & 17,9 \\
\hline
\end{tabular}

\section{Factores de riesgo para el consumo de alcohol}

La media de edad para el consumo episódico excesivo fue de 20,5 años. La media de edad para el no consumo episódico excesivo de alcohol fue de 20,3 años. La diferencia de medias de edad en el consumo episódico excesivo de alcohol fue de $(-0,2) p=0,01$. La media de edad de inicio de consumo de alcohol para quienes poseían consumo episódico excesivo fue de 15,2 años, la media de edad de inicio de consumo de alcohol para quienes no poseían consumo episódico excesivo fue de 15,87 años. La diferencia de medias para la edad a la que consumió alcohol por primera vez fue de 0,66 p<0,001.

En la Tabla 4 se resumen las variables categóricas relacionadas al consumo episódico excesivo de alcohol. Existe mayor riesgo de consumo episódico excesivo de alcohol en adolescentes y jóvenes que viven en la región Noroeste, Noreste y Patagónica, en relación con la región de Gran Buenos Aires, la existencia de dos o más indicadores NBI y ser de género masculino. Como factores protectores se encuentran, los adolescentes y jóvenes que provienen de hogares multipersonales completo sin hijos, en relación con los que provienen de hogares unipersonales, tener jefe/a de hogar entre 25 y 34 años en relación a jefes/as de hogar menores de 25 años, provenir de un hogar con jefe/a de hogar casado en comparación a jefe/a de hogar soltero/a, provenir de un hogar con jefatura de hogar que posea educación secundaria completa o más; ser adolescente cónyuge o pareja, o hijo/a en relación a ser jefe/a de hogar; ser adolescente o joven inactivo/a, no haber tenido accidente en los últimos doce meses, no haber tenido curiosidad de probar alguna droga, no haber tenido posibilidad de probar drogas alguna vez, no sentir deseos de probar drogas, y percepción de que existe un gran riesgo acerca de ser un bebedor frecuente. 
Tabla 4 - Factores de riesgo y Protección para el consumo episódico excesivo de alcohol en adolescentes y jóvenes de 16 a 24 años de Argentina. Encuesta EnPreCoSP-2008

\begin{tabular}{|c|c|c|c|c|c|}
\hline \multirow{2}{*}{ Región } & \multirow{2}{*}{$\begin{array}{l}\text { Categorías } \\
\text { Referencia: Gran Buenos Aires }\end{array}$} & \multirow[t]{2}{*}{ Sig. } & \multirow[t]{2}{*}{ OR } & \multicolumn{2}{|c|}{ IC } \\
\hline & & & & & \\
\hline & Pampeana & 0,134 & 1,301 & 0,922 & 1,837 \\
\hline & Noroeste & 0,000 & 1,952 & 1,390 & 2,741 \\
\hline & Noreste & 0,005 & 1,657 & 1,165 & 2,355 \\
\hline & Cuyo & 0,821 & 0,958 & 0,661 & 1,390 \\
\hline & Patagónica & 0,038 & 1,444 & 1,020 & 2,045 \\
\hline \multirow[t]{8}{*}{ Tipo de hogar } & Referencia: Unipersonal & & & & \\
\hline & Multipersonal conyugal completo sin hijos ni otros miembros & 0,005 & 0,450 & 0,259 & 0,782 \\
\hline & Multipersonal conyugal completo sin hijos y con otros miembros & 0,624 & 0,850 & 0,444 & 1,628 \\
\hline & Multipersonal conyugal completo con hijos y sin otros miembros & 0,076 & 0,773 & 0,581 & 1,028 \\
\hline & Multipersonal conyugal completo con hijos y con otros miembros & 0,990 & 1,002 & 0,721 & 1,393 \\
\hline & Multipersonal conyugal incompleto sin otros miembros & 0,779 & 1,046 & 0,765 & 1,429 \\
\hline & Multipersonal conyugal incompleto con otros miembros & 0,798 & 0,956 & 0,677 & 1,350 \\
\hline & Multipersonal no conyugal & 0,509 & 0,892 & 0,635 & 1,253 \\
\hline \multirow[t]{4}{*}{ Necesidades Básicas Insatisfechas } & Referencia: Ningún indicador NBI & & & & \\
\hline & Al menos un indicador NBI & 0,756 & 0,845 & 0,293 & 2,439 \\
\hline & Al menos dos indicadores NBI & 0,000 & 1,656 & 1,328 & 2,064 \\
\hline & Tres o más indicadores NBI & 0,000 & 2,383 & 1,557 & 3,649 \\
\hline \multirow[t]{5}{*}{ Grupo de edad del jefe/a } & Referencia: 16 a 24 años & & & & \\
\hline & 25 a 34 años & 0,000 & 0,515 & 0,360 & 0,737 \\
\hline & 35 a 49 años & 0,214 & 0,885 & 0,729 & 1,073 \\
\hline & 50 a 65 años & 0,108 & 0,849 & 0,695 & 1,036 \\
\hline & 66 años y más & 0,183 & 0,813 & 0,599 & 1,103 \\
\hline \multirow[t]{6}{*}{ Situación conyugal del jefe/a } & Referencia: Soltero/a & & & & \\
\hline & Unido/a & 0,625 & 0,945 & 0,752 & 1,186 \\
\hline & Casado/a & 0,003 & 0,743 & 0,612 & 0,902 \\
\hline & Separado/a & 0,284 & 1,158 & 0,885 & 1,515 \\
\hline & Divorciado/a & 0,217 & 0,792 & 0,547 & 1,147 \\
\hline & Viudo/a & 0,168 & 0,812 & 0,603 & 1,092 \\
\hline \multirow[t]{8}{*}{ Nivel de instrucción del jefe } & Referencia: Sin instrucción & & & & \\
\hline & Primario incompleto & 0,322 & 0,747 & 0,419 & 1,331 \\
\hline & Primario completo & 0,177 & 0,681 & 0,390 & 1,190 \\
\hline & Secundario incompleto & 0,248 & 0,716 & 0,406 & 1,262 \\
\hline & Secundario completo & 0,039 & 0,553 & 0,315 & 0,970 \\
\hline & Terciario o universitario incompleto & 0,023 & 0,519 & 0,295 & 0,915 \\
\hline & Terciario o universitario completo y más & 0,002 & 0,399 & 0,222 & 0,718 \\
\hline & Educación especial & 1,000 & 0,000 & 0,000 & . \\
\hline \multirow{9}{*}{$\begin{array}{l}\text { Relación del parentesco con el } \\
\text { jefe/a }\end{array}$} & Referencia: Jefe/a & & & & \\
\hline & Cónyuge/pareja & 0,000 & 0,338 & 0,224 & 0,512 \\
\hline & Hijo/a / hijastro/a & 0,032 & 0,807 & 0,664 & 0,982 \\
\hline & Hermano/a & 0,248 & 0,776 & 0,505 & 1,193 \\
\hline & Suegro/a & 0,999 & 0,000 & 0,000 & . \\
\hline & Yerno/nuera & 0,843 & 0,944 & 0,534 & 1,670 \\
\hline & Nieto/a & 0,120 & 0,750 & 0,522 & 1,078 \\
\hline & Otro familiar & 0,086 & 0,632 & 0,374 & 1,067 \\
\hline & Otro no familiar & 0,717 & 1,092 & 0,678 & 1,760 \\
\hline \multirow[t]{2}{*}{ Sexo } & Referencia: Mujer & & & & \\
\hline & Hombre & 0,000 & 3,808 & 3,238 & 4,478 \\
\hline \multirow[t]{3}{*}{ Condición de actividad } & Referencia: Ocupado & & & & \\
\hline & Desocupado & 0,611 & 0,934 & 0,717 & 1,216 \\
\hline & Inactivo & 0,000 & 0,563 & 0,483 & 0,657 \\
\hline \multirow[t]{3}{*}{ Accidente en los últimos 12 meses } & Referencia: Sí & & & & \\
\hline & No & 0,000 & 0,613 & 0,477 & 0,787 \\
\hline & $\mathrm{Ns} / \mathrm{Nc}$ & 1,000 & 0,000 & 0,000 & . \\
\hline
\end{tabular}


Tabla 4 - (continuación)

\begin{tabular}{|c|c|c|c|c|c|}
\hline Variable & Categorías & Sig. & OR & & \\
\hline \multirow{4}{*}{$\begin{array}{l}\text { Sintió alguna vez curiosidad por } \\
\text { probar alguna droga }\end{array}$} & Referencia: Sí & & & & \\
\hline & no & 0,000 & 0,381 & 0,322 & 0,451 \\
\hline & Puede ser & 0,062 & 0,571 & 0,317 & 1,028 \\
\hline & $\mathrm{Ns} / \mathrm{Nc}$ & 0,535 & 0,621 & 0,138 & 2,794 \\
\hline \multirow{3}{*}{$\begin{array}{l}\text { Posibilidad de probar drogas } \\
\text { alguna vez }\end{array}$} & Sí & & & & \\
\hline & No & 0,000 & 0,478 & 0,414 & 0,553 \\
\hline & $\mathrm{Ns} / \mathrm{Nc}$ & 0,117 & 0,356 & 0,098 & 1,297 \\
\hline \multirow[t]{4}{*}{ Probaría alguna } & Referencia: Sí & & & & \\
\hline & No & 0,000 & 0,222 & 0,169 & 0,292 \\
\hline & Puede ser & 0,000 & 0,355 & 0,232 & 0,544 \\
\hline & $\mathrm{Ns} / \mathrm{Nc}$ & 0,000 & 0,277 & 0,149 & 0,513 \\
\hline \multirow{4}{*}{$\begin{array}{l}\text { Percepción acerca del riesgo de un } \\
\text { bebedor frecuente }\end{array}$} & Referencia: Ningún riesgo & & & & \\
\hline & Riesgo leve o moderado & 0,208 & 0,576 & 0,245 & 1,358 \\
\hline & Gran riesgo & 0,008 & 0,323 & 0,141 & 0,741 \\
\hline & No sé qué riesgo corre & 0,571 & 0,756 & 0,287 & 1,990 \\
\hline
\end{tabular}

Existió relación entre el consumo episódico excesivo de alcohol y la prevalencia de vida de consumo de tabaco $\mathrm{OR}=2,4(2,0-2,7 \mathrm{p}<0,001)$; consumo de tranquilizantes $\mathrm{OR}=1,9(1,4-2,6 \mathrm{p}<0,001)$; consumo de estimulantes $\mathrm{OR}=2,2(1,18-4,2 \mathrm{p}=0,02)$; consumo de marihuana $\mathrm{OR}$ $=3,4(2,8-4,2 \mathrm{p}<0,001)$; consumo de cocaína $\mathrm{OR}=9,1$ (5,5-15,3 $\mathrm{p}<0,001)$; consumo de pasta base OR 41,9 $(5,7-310,0 \mathrm{p}<0,001)$; consumo de éxtasis OR 5,2 (2,1$13,1 \mathrm{p}<0,001)$; consumo de inhalables OR 4,8 (3,0-7,8 $\mathrm{p}<0,001)$; consumo de opiáceos y anestésicos OR 3,2 $(1,3-8,0 \mathrm{p}=0,01)$; consumo de alucinógenos $\mathrm{OR}=5,2$ $(2,2-12,3 p<0,001)$.
En el análisis multivariado se encontró como factores de riesgo la edad de inicio (a menor edad, mayor el riesgo) la región Pampeana, Noroeste y Noreste, ser hombre y provenir de hogares con NBI. Entre los factores protectores se encuentra encontrarse inactivo, no haber tenido posibilidad de probar drogas ilícitas, no sentir deseos de probar drogas ilícitas: y no haber consumido tabaco, ni tranquilizantes, ni cocaína, ni pasta base alguna vez en la vida (Tabla 5). La edad de inicio también fue un factor de riesgo, a menor edad de inicio, mayor es el riesgo ( $\mathrm{OR}=0,88 \mathrm{IC}=0,84-0,92$. $p<0,001)$.

Tabla 5 - Factores de riesgo y Protección para el consumo episódico excesivo de alcohol en adolescentes y jóvenes de 16 a 24 años de Argentina. Encuesta EnPreCoSP-2008

\begin{tabular}{|c|c|c|c|c|c|}
\hline Variable & Categorías & Sig. & OR & \multicolumn{2}{|c|}{ IC } \\
\hline \multirow{5}{*}{ Región } & Pampeana & 0,02 & 1,64 & 1,09 & 2,46 \\
\hline & Noroeste & 0,00 & 2,65 & 1,77 & 3,98 \\
\hline & Noreste & 0,00 & 2,44 & 1,60 & 3,71 \\
\hline & Cuyo & 0,26 & 1,29 & 0,83 & 1,99 \\
\hline & Patagónica & 0,06 & 1,49 & 0,99 & 2,25 \\
\hline \multirow[t]{2}{*}{ Sexo } & Referencia: Mujer & & & & \\
\hline & Hombre & 0,00 & 3,31 & 2,75 & 4,00 \\
\hline \multirow[t]{4}{*}{ Necesidades Básicas Insatisfechas } & Referencia: Ningún indicador NBI & & & & \\
\hline & Al menos un indicador NBI & 0,48 & 0,62 & 0,17 & 2,31 \\
\hline & Al menos dos indicadores $\mathrm{NBI}$ & 0,02 & 1,39 & 1,06 & 1,84 \\
\hline & Tres o más indicadores NBI & 0,04 & 1,74 & 1,04 & 2,90 \\
\hline \multirow[t]{3}{*}{ Condición de actividad } & Referencia: Ocupado & & & & \\
\hline & Desocupado & 0,49 & 1,11 & 0,82 & 1,52 \\
\hline & Inactivo & 0,02 & 0,80 & 0,65 & 0,97 \\
\hline
\end{tabular}


Tabla 5 - (continuación)

\begin{tabular}{|c|c|c|c|c|c|}
\hline $\begin{array}{c}\text { Variable } \\
\text { Posibilidad de probar drogas alguna vez }\end{array}$ & Categorías & Sig. & OR & \multicolumn{2}{|c|}{ IC } \\
\hline & No & 0,00 & 0,74 & 0,61 & 0,89 \\
\hline & $\mathrm{Ns} / \mathrm{Nc}$ & 0,15 & 0,33 & 0,07 & 1,52 \\
\hline \multirow[t]{4}{*}{ Probaría alguna } & Referencia: Sí & & & & \\
\hline & No & 0,00 & 0,57 & 0,39 & 0,83 \\
\hline & Puede ser & 0,05 & 0,61 & 0,37 & 1,00 \\
\hline & $\mathrm{Ns} / \mathrm{Nc}$ & 0,09 & 0,52 & 0,24 & 1,12 \\
\hline \multirow[t]{2}{*}{ Prevalencia de vida de consumo de tabaco } & Referencia: Sí & & & & \\
\hline & No & 0,00 & 0,59 & 0,49 & 0,72 \\
\hline \multirow[t]{2}{*}{ Prevalencia de vida de consumo de tranquilizantes } & Referencia: Sí & & & & \\
\hline & No & 0,00 & 0,53 & 0,35 & 0,79 \\
\hline \multirow[t]{2}{*}{ Prevalencia de vida de consumo de cocaína } & Referencia: Sí & & & & \\
\hline & No & 0,00 & 0,38 & 0,20 & 0,70 \\
\hline \multirow[t]{2}{*}{ Prevalencia de vida de consumo de pasta base } & Referencia: Sí & & & & \\
\hline & No & 0,05 & 0,11 & 0,01 & 0,99 \\
\hline
\end{tabular}

\section{Discusión}

En relación a la prevalencia de consumo episódico excesivo de alcohol en adolescentes (Tabla 1) es similar al reportado por países Europeos $(18 \%)^{(11)}$, y en los Estados Unidos $(19 \%)^{(12)}$. En Latinoamérica, se ha descrito un prevalencia de consumo episódico excesivo de alcohol que va desde el 6,7 \% hasta el 32,4\%(13-14). Sin embargo, los datos en relación a la prevalencia de consumo poseen una alta proporción (40\%) sin datos, por lo cual los valores de adolescentes y jóvenes que presentan consumo episódico excesivo podrían ser mayores.

Un estudio previo de consumo de alcohol en Argentina(15) reflejó un porcentaje similar (18\%) en el consumo episódico excesivo de alcohol, de la misma manera, la bebida más consumida fue la cerveza, en especial los fines de semana. Por otra parte, también se ha reportado un mayor consumo en jóvenes del Noroeste del país. La relación entre el consumo episódico excesivo y la región geográfica del país, podría estar fundamentado en las diferencias entre las condiciones de vida entre regiones, ya que las regiones Noroeste $y$ Noreste son las que poseen mayores índices de pobreza. Asimismo, el presente estudio ha observado relación entre el consumo episódico excesivo y la pobreza estructural, reflejada por el indicador NBI, diversos estudios también han reportado la relación entre el consumo y el bajo nivel socioeconómico(14,16-19). En el análisis multivariado, al comparar con otras variables, la región Pampeana también presenta riesgo significativo de consumo episódico excesivo en jóvenes (Tabla 5).

Se destaca en los resultados, la percepción de riesgo de consumo excesivo de alcohol, por parte de los adolescentes y jóvenes. La percepción de ser un bebedor excesivo "de vez en cuando", no es vista como de gran riesgo por la mayoría de los jóvenes, aunque si es vista como de riesgo por la mayoría de la población, el ser bebedor frecuente; e incluso es un factor protector para el consumo episódico excesivo de alcohol.

En relación al género, se destaca como en otros estudios que existió mayor riesgo de consumo episódico excesivo en el sexo masculino, igualmente en relación a la edad de inicio, en donde se observó mayor riesgo a menor edad de inicio de consumo de alcohol $\left.\right|^{(1-3,6-7,9,11}$ $16,20-23)$

En cuanto a las características del jefe de hogar, se observó relación significativa, con el tipo de hogar, estos resultados concuerdan con los relatados por otros autores $^{(17-18)}$, los cuales observaron relación entre la mala estructura familiar y el riesgo de consumo episódico excesivo.

En cuanto a las características del jefe/a de hogar, nivel educativo y la situación conyugal del jefe de hogar, se destaca que en la mayor parte de la población el jefe/a de hogar es el padre/madre del adolescente/ joven; y en segundo lugar el propio adolescente/joven. Estudios previos(17-18), han observado relación entre 
factores psicosociales y el consumo excesivo de alcohol, entre los cuales se encontraban la estructura marital y el nivel de educacion de los padres y/o adolescentes/ jóvenes.

En cuanto a la condición de actividad, se observó un factor de protección el hecho del adolescente encontrarse inactivo, es decir que no tenía trabajo y tampoco lo buscaba. Esto podría explicarse por la inserción en el sistema educativo, lo cual es un factor protector, anteriormente descrito. Por otra parte, al encontrarse inactivos, disponen menos cantidad de dinero; estudios previos destacan que existe mayor riesgo de consumo de sustancias psicoactivas, entre ellas el alcohol, cuando el adolescente posee mayor disponibilidad de dinero(20).

Entre las variables relacionadas con la salud del adolescente se observó que el haber sufrido accidente en el último año, estuvo asociado con el consumo episódico excesivo de alcohol. Está variable se podría considerar más una consecuencia del consumo excesivo que un factor de riesgo ${ }^{(16-21)}$.

Se observó que el consumo episódico excesivo tuvo relación con el acceso a sustancias psicoactivas ilícitas de los/as adolescentes así como el consumo de otras sustancias psicoactivas, esta relación reportada por otros estudios ${ }^{(9-22)}$.

\section{Conclusiones}

Los resultados del presente artículo indican que el consumo excesivo de alcohol es un patrón de consumo frecuente en los/las adolescentes y jóvenes. El conocimiento de los factores de riesgo, aporta información útil para la formulación de programas de prevención, enfatizando la atención a las poblaciones que poseen mayor riesgo de este comportamiento; así como promover factores protectores en la población general.

\section{Agradecimientos}

Agradecemos a la Comisión Interamericana para el Control del Abuso de Drogas/CICAD de la Secretaria de Seguridad Multidimensional/SSM de la Organización de los Estados Americanos/OEA, la Secretaria Nacional de Políticas sobre Drogas/SENAD do Gabinete de Seguridad Institucional/Brasil, la Escuela de Enfermería de Ribeirao Preto de la Universidad de Sao Paulo y Centro Colaborador de la Organización Mundial de la Salud para el Desarrollo de la Investigación en Enfermería, la población representada en los estudios de investigación, bien como a las autoridades de las universidades representadas por los participantes del Programa En-Line de Especialización en Investigación sobre el Fenómeno de las Drogas, periodos 2006, 2007, 2008 y 2009.

\section{Referencias}

1. Brown SA, McGue M, Maggs J, Schulenberg J, Hingson $\mathrm{R}$, Swartzwelder $\mathrm{S}$, et al. A developmental perspective on Alcohol and Youths 16 to 20 years of age. Pediatrics. 2008; 121(suppl 4):S290-S310.

2. Hawkins JD, Catalano RF, Miller JF. Risk and protective factors for alcohol and other drug problems in adolescence and early adulthood: Implications for substance abuse prevention. Psychol Bull. 1992;112:64-105.

3. Oliveira MR, Luís MAV. Factores de riesgo para el consumo de alcohol en escolares de 10 a 18 años de establecimientos educativos fiscales de la ciudad de la Paz Bolivia (2003-2004) Rev. Latino-Am. Enfermagem. 2005;13(n० esp):880-7.

4. Míguez $\mathrm{H}$. Usuarios de drogas y delito. Datos de la provincia de Buenos Aires, Argentina. VERTEX. 2009;20(83):35-9.

5. Leifman $H$, Österberg $E$, Ramstedt $M$. Alcohol in Postwar Europe, ECAS II: A discussion of indicators and alcohol-related harm. Stockholm: Almquist and Wiksell International; 2002.

6. Wechsler $\mathrm{H}$, Nelson TF. Binge drinking and the American college student: What's five/four drinks? Psychol Addict Behav. 2001;15:287-91.

7. Miller JW, Naimi TS, Brewer RD, Jones SE. Binge drinking and associated health risk behaviors among high school students. Pediatrics. 2007;119:76-85.

8. Puddey IB, Rakic V, Dimmitt SB, Beilin LJ. Influence of pattern of drinking on cardiovascular disease and cardiovascular risk factors: a review. Addiction. 1999;94:649-3.

9. Wechsler H, Davenport A, Dowdall G. Health and behavioral consequences of binge drinking in college. A national survey of students at 140 campuses. JAMA. $1994 ; 272: 1672-7$

10. Encuesta Nacional sobre Prevalencia de Consumo de Sustancias Psicoactivas (INDEC EnPreCoSP 2008) (AR). Documento para la utilización de Base de Datos Usuario. Instituto Nacional de Estadísticas y Censos. Buenos Aires: Ministerio de Salud de la Nación; 2009.

11. Farke W, Anderson P. El consumo concentrado de alcohol en Europa. Adicciones. 2007; 19(4):330-40.

12. Centers for Disease Control and Prevention (CDC). Alcohol Use among high school students Georgia 2007. JAMA. 2010;303(1):30-1. 
13. Martin KK, Loveland-Cherry CJ, Villaroel AM. Mexicans adolescents' alcohol use, family intimacy and parent-adolescent communication. J Family Nurs. 2009;15(2):152-70.

14. Silveira CM, Silveira CC, Silva JG, Silveira LM, Andrade AG, Andrade LHSG. Epidemiologia do beber pesado episódico no Brasil: uma revisão sistemática da literatura. Rev Psiq Clin. 2008;35(1):31-8.

15. Observatorio Argentino de Drogas. Estudio Nacional en Población de 12 a 65 años sobre Consumo de sustancias Psicoactivas. Argentina, 2006. Informe de Resultados; 2007.

16. Stolle M, Sack PM, Thomasius R. Binge Drinking in Childhood and Adolescence. Epidemiology, Consequences and Interventions. Dtsch Arztebl Int. 2009;106(9):323-8.

17. Yang S, Lynch JW, Raghunathan TE, Kauhanen J, Salonen J, Kaplan GA. Socioeconomic and Psychosocial Exposures across the Life Course and Binge Drinking in Adulthood: Population Based Study. Am J Epidemiol. 2007;(165): 184-93.

18. Herrera Santi P. Principales factores de riesgo psicológicos y sociales en el adolescente. Rev Cub Pediatr. $1999 ; 71(1): 39-42$.

19. National Institute on Drug Abuse. Preventing Drug Abuse among Children and Adolescents. A ResearchBased Guide for Parents, Educators and Community Leaders. 2.ed. USA: U.S. Department of Health and Human Services. National Institute of Health; 2003.

20. Burrone MS, Villela SMV, Costa ML, Enders J, Fernández AR, Vasters GP. Análisis de la frecuencia de experimentación y consumo de drogas de alumnos de escuelas del nivel medio. Rev. Latino-Am. Enfermagem. 2010;18(no esp):648-54.

21. Ramchand R, Marshall GN, Schell TN, Jaycox L, Hambarsoomians K, Shetty $V$, et al. Alcohol abuse and illegal drug use among Los Angeles County trauma patients: prevalence and evaluation of single item screener. J Trauma. 2009;66(5):1461-7.

22. Jones SE, Oeltmann J, Wilson TW, Brener ND, Hill CV. Binge drinking among undergraduate college students in the United States: implications for other substance use. J Am Coll Health. 2001;50(1):33-8.

23. Courtney KE, Polich J. Binge Drinking in Young Adults: data, Definitions and Determinants. Psychol

Recibido: 3.3 .2011 Bull. 2009;135(1):142-56. 